\title{
Identification of 20(S)-Ginsenoside Rh2 as a Potential EGFR Tyrosine Kinase Inhibitor
}

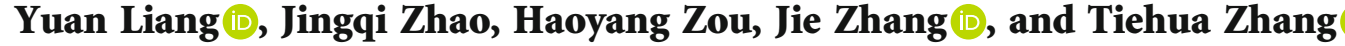 \\ College of Food Science and Engineering, Jilin University, Changchun 130062, China \\ Correspondence should be addressed to Tiehua Zhang; zhangth@jlu.edu.cn
}

Received 27 August 2021; Revised 29 October 2021; Accepted 21 December 2021; Published 24 January 2022

Academic Editor: ChongDe Sun

Copyright (c) 2022 Yuan Liang et al. This is an open access article distributed under the Creative Commons Attribution License, which permits unrestricted use, distribution, and reproduction in any medium, provided the original work is properly cited.

\begin{abstract}
As the main active ingredients of Panax ginseng, ginsenosides possess numerous bioactivities. Epidermal growth factor receptor (EGFR) was widely used as a valid target in anticancer therapy. Herein, the EGFR targeting activities of 20(S)-ginsenoside Rh2 $(20(S)-\mathrm{Rh} 2)$ and the relationship of their structure-activity were investigated. Homogeneous time-resolved fluorescence assay showed that 20(S)-Rh2 significantly inhibited the activity against EGFR kinase. 20(S)-Rh2 was confirmed to effectively inhibited cell proliferation in a dose-dependent manner by MTT assay. Furthermore, quantitative real-time PCR and western blotting analysis revealed that 20(S)-Rh2 inhibited A549 cells growth via the EGFR-MAPK pathway. Meanwhile, 20(S)-Rh2 could promote cell apoptosis, block cell cycle, and reduce cell migration of A549 cells, respectively. In silico, the result suggested that both hydrophobic interactions and hydrogen-bonding interactions could contribute to stabilize their binding. Molecular dynamics simulation showed that the side chain sugar moiety of $20(S)$-Rh2 was too flexible to be fixed at the active site of EGFR. Collectively, these findings suggested that 20(S)-Rh2 might serve as a potential EGFR tyrosine kinase inhibitor.
\end{abstract}

\section{Introduction}

Lung cancer is the leading cause of cancer death worldwide. Non-small-cell lung cancer (NSCLC) contributes over $80 \%$ of lung cancer cases with a low 5-year survival rate [1]. The development of epidermal growth factor receptortyrosine kinase inhibitors (EGFR-TKIs) plays a key role in the targeted therapy of NSCLC. Epidermal growth factor receptor (EGFR) is considered a receptor tyrosine kinase with penetrating the cell membrane [2]. EGFR is composed of an extracellular ligand-binding region, a transmembrane region, and an intracellular tyrosine kinase region [3]. Binding to the ligand, EGFR forms a dimer and the phosphate of ATP transfers into the tyrosine residue. Then, different kinds of proteins bind to this phosphorylated tyrosine and signals transmit to downstream pathways, such as mitogen-activated protein kinase (MAPK) and phosphatidylinositol 3-kinase (PI3K) pathways $[4,5]$. It is well known that EGFR plays a key role in cell proliferation, apoptosis, and migration [6]. Furthermore, EGFR is confirmed to be dysregulated or overexpressed in various solid tumors and used as one of the valid targets in anticancer therapy $[7,8]$.

As the main active ingredients of Panax ginseng (P. ginseng), ginsenosides have been widely used in cancer treatment with reduced side effects [9-11]. Ginsenoside Rh2 can be divided into 20(S)-ginsenoside $\mathrm{Rh} 2(20(S)$ - $\mathrm{Rh} 2)$ and 20(R)-ginsenoside $\mathrm{Rh} 2(20(R)-\mathrm{Rh} 2)$ according to different orientations of the hydroxyl group at C-20 position [12, 13]. Tumor-associated macrophages (TAMs) are confirmed to play crucial roles in modulating the tumor microenvironment and promoting tumor metastases [14]. Rh2 showed potential to convert TAMs from the alternatively activated M2 macrophages to classically activated M1 macrophages in the microenvironment. Meanwhile, Rh2 prevented NSCLC cell migration, suggesting the therapeutic effects of $\mathrm{Rh} 2$ on lung cancer [15]. Rh2 was also demonstrated to inhibit the proliferation and metastasis of NSCLC cells by inducing apoptosis and suppressing epithelialmesenchymal transition, respectively [16]. In the chemotherapy for NSCLC patients, Rh2 enhanced the antitumor effects of cisplatin through inhibiting the superoxide 
generation, PD-L1 expression, and cell autophagy [17]. However, the mechanism of 20(S)-Rh2 targeting EGFR to inhibit cell proliferation is not clear enough now.

Hence, this work is aimed at identifying $20(S)-\mathrm{Rh} 2$ as a potential EGFR tyrosine kinase inhibitor by the combination of in vitro and in silico approaches. Homogeneous timeresolved fluorescence (HTRF) assay was performed to detect the EGFR kinase activity after the treatment of 20(S)-Rh2. Cell viability was measured by MTT assay. To investigate whether 20(S)-Rh2 regulated the EGFR-MAPK pathway, the changes in gene expressions and protein contents were determined by quantitative real-time PCR and western blot analysis, respectively. Meanwhile, cell apoptosis and cycle analyses were taken by flow cytometry. Cell wound healing assay was also performed to measure the migration of A549 cells. On this basis, the possible binding conformation of $20(S)-\mathrm{Rh} 2$ with EGFR was predicted using molecular docking. The binding stability of the EGFR-20(S)-Rh2 complex was explored using molecular dynamics simulation.

\section{Materials and Methods}

2.1. Materials. $\mathrm{HTRF}^{\circledR}$ KinEASE-TK assay kit was obtained from Cisbio (Codolet, France). EGFR protein (GST-tagged) was obtained from Thermo Fisher Scientific (Carlsbad, CA, USA). Dulbecco's modified Eagle's medium (DMEM, low glucose), penicillin and streptomycin, trypsin, and phosphate buffer saline (PBS) were obtained from Gibco (Paisley, UK). Fetal bovine serum (FBS) was obtained from Zhejiang Tianhang Biotechnology Co., Ltd. (Zhejiang, Hangzhou, China). 3-(4,5-Dimethylthiazol-2-yl)-2,5-diphenyltetrazolium bromide (MTT) was obtained from Shanghai Aladdin Biochemical Technology Co., Ltd. (Shanghai, China). 20(S)-Rh2 and bovine serum albumin (BSA) were obtained from Yuanye Biotech Co., Ltd. (Shanghai, China). Dimethyl sulfoxide (DMSO) was obtained from Beijing Solarbio Science \& Technology Co., Ltd. (Beijing, China). Ethylenediamine tetraacetic acid (EDTA) was obtained from SigmaAldrich (MO, USA). Goat anti-rat IgG secondary antibody was obtained from Sino Biological Inc. (Beijing, China). Antibody against GAPDH was obtained from Gene Tex (Irvine, CA, USA). Other primary antibodies were all obtained from Abcam (Cambridge, MA, USA). All other chemical reagents were of analytical grade.

2.2. Measurement of EGFR Kinase Activity by 20(S)-Rh2. The enzyme reaction was conducted for $40 \mathrm{~min}$ at room temperature in white HTRF 96-well low volume plate. 20(S)-Rh2 was 10 -fold serial dilutions from $10^{3}$ to $10^{-3} \mu \mathrm{M}$. During the enzymatic step, $20(S)-\mathrm{Rh} 2(4 \mu \mathrm{L})$ and the TK Substrate-biotin $(50 \mu \mathrm{M}, 2 \mu \mathrm{L})$ were incubated with the EGFR kinase ( $41 \mathrm{nM}, 2 \mu \mathrm{L})$; then, ATP $(1.41 \mu \mathrm{M}, 2 \mu \mathrm{L})$ was added to start the reaction. The enzymatic buffer contained $5 \mathrm{mM} \mathrm{MgCl}_{2}, 1 \mathrm{mM} \mathrm{MnCl}$, and $1 \mathrm{mM}$ DTT. Subsequently, at the detection step, streptavidin-XL665 (16.67 $\mu \mathrm{M}, 5 \mu \mathrm{L})$ and TK-antibody labeled with $\mathrm{Eu}^{3+}$-cryptate $(5 \mu \mathrm{L})$ in the detection buffer with EDTA were added to stop the kinase activity. After incubation for another $1 \mathrm{~h}$ at room temperature, fluorescence was measured at both $620 \mathrm{~nm}$ and
$665 \mathrm{~nm}$ with a microplate reader (Tecan Spark $^{\circledR} 10 \mathrm{M}$, Tecan, Männedorf, Switzerland) [18, 19]. The half maximal inhibitory concentration $\left(\mathrm{IC}_{50}\right)$ was calculated by PRISM version 5.0 software (GraphPad Software Inc., CA, USA).

2.3. Cell Culture. The human non-small-cell lung cancer cell line A549 was obtained from Cell Bank of Chinese Academy of Sciences (Shanghai, China). A549 cells were cultured in the DMEM supplemented with $10 \%$ FBS, $100 \mathrm{U} / \mathrm{mL}$ penicillin, and $0.1 \mathrm{mg} / \mathrm{mL}$ streptomycin, at $37^{\circ} \mathrm{C}$ in a humidified atmosphere of $5 \% \mathrm{CO}_{2}$.

2.4. MTT Assay. A549 cells were seeded in 96-well plates at a density of $1 \times 10^{4}$ cells per well and incubated for $24 \mathrm{~h}$ to allow for cell attachment. Then, A549 cells were incubated with different concentrations $(5,10,20,30,40,50,60,80$, 100 , and $120 \mu \mathrm{M})$ of $20(S)-\mathrm{Rh} 2$ for another $24 \mathrm{~h}$. The culture medium was removed and replaced with new culture medium with MTT solution $(5 \mathrm{mg} / \mathrm{mL})$ and incubated at $37^{\circ} \mathrm{C}$ for $4 \mathrm{~h}$. Then, the medium was removed and DMSO $(110 \mu \mathrm{L})$ was added to each well to dissolve the crystals. The absorbance at $490 \mathrm{~nm}$ was measured with a microplate reader (Bio-Rad, Hercules, CA, USA). The cell viabilities of A549 cells were calculated as the percentage of absorbance compared to DMSO control cells.

2.5. Quantitative Real-Time PCR. A549 cells were seeded in $10 \mathrm{~cm}$ discs at a density of $1 \times 10^{6}$ cells per disc and incubated overnight; then, cells were incubated with different concentrations $(15,22$, and $35 \mu \mathrm{M})$ of $20(S)$-Rh2 for another $24 \mathrm{~h}$. Total RNA was extracted using TRIzol reagent (Transgen Biotech Co., Ltd., Beijing, China). Reverse transcription and PCR amplification were carried out with TransScript one-step gDNA removal and cDNA synthesis supermix kit (Transgen Biotech Co., Ltd., Beijing, China). Quantitative real-time PCR was conducted using $\mathrm{CFX} 96^{\mathrm{TM}}$ real-time system (Bio-rad ${ }^{\circledR}$, Hercules, CA, USA) along with the primer pairs listed in the past article of our team [20]. The relative expression levels of mRNA were calculated by the $2^{-\Delta \Delta C T}$ method normalized with the GAPDH RNA level.

2.6. Western Blotting Analysis. A549 cells were seeded in $10 \mathrm{~cm}$ discs at a density of $5 \times 10^{6}$ cells per disc and incubated for $24 \mathrm{~h}$; then, cells were incubated with different concentrations $(15,22$, and $35 \mu \mathrm{M})$ of $20(S)-\mathrm{Rh} 2$ for another $24 \mathrm{~h}$. Cell samples were washed with the ice-cold PBS and lysed with RIPA buffer (Cell Signaling Technology, Danvers, MA, USA) containing protease/phosphatase inhibitors (Cell Signaling Technology, Danvers, MA, USA). Cell protein lysates were separated by SDS-polyacrylamide gel electrophoresis (PAGE) and then transferred onto a polyvinylidene difluoride (PVDF) membrane (Millipore, USA). After being blocked with 5\% skim milk or BSA dissolved in TBST buffer, the membranes were incubated with primary antibodies at $4^{\circ} \mathrm{C}$ overnight. Then, goat anti-rat IgG secondary antibody was added. Following the washing step of the TBST buffer, an ECL immunoblotting detection kit (Clinx Science Instrument Co., Ltd., Shanghai, China) was used to detect the visualization. Antibody against GAPDH was used as an internal control. 


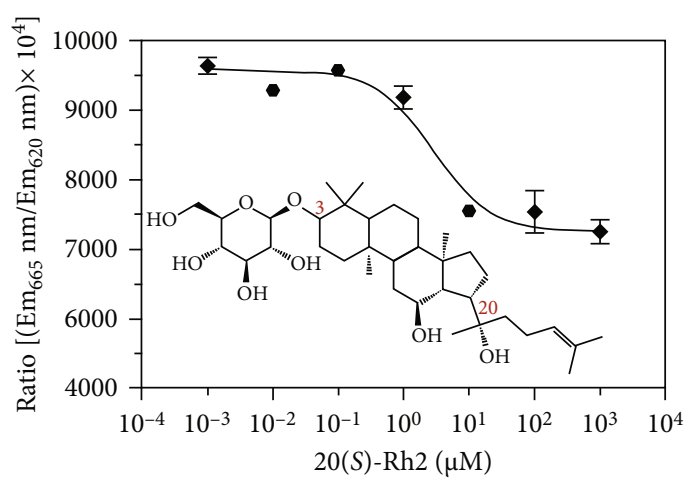

(a)

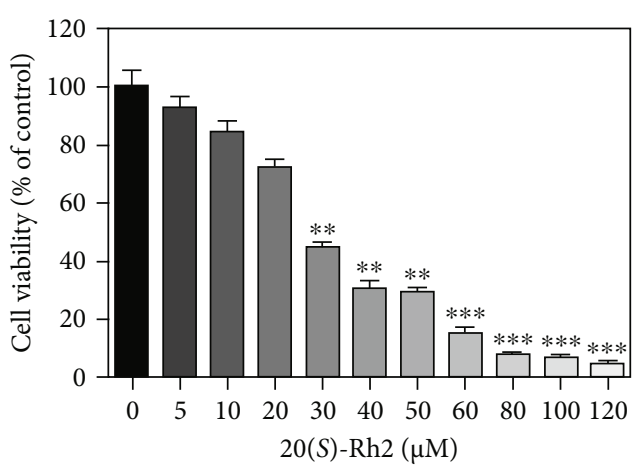

(b)

FIGURE 1: (a) 20(S)-Rh2 inhibited the activity of EGFR kinase and (b) A549 cell proliferation. DMSO-treated group was used as control. ${ }^{*} p<0.05,{ }^{* *} p<0.01$, and ${ }^{* * *} p<0.001$.

2.7. Analysis of Cell Apoptosis and Cycle by Flow Cytometry. A549 cells were seeded in 6-well plates at a density of 2.5 $\times 10^{5}$ cells per well and incubated for $24 \mathrm{~h}$; then, cells were incubated with different concentrations (15, 22, and $35 \mu \mathrm{M})$ of $20(S)$-Rh2 for another $24 \mathrm{~h}$. A549 cells were harvested with EDTA-free trypsin and washed with the icecold PBS. Then, cell apoptosis analysis was performed using annexin V-FITC/PI apoptosis detection kit (Meilunbio, Dalian, China). Followed by centrifugation at $1000 \mathrm{~g}$ for $5 \mathrm{~min}$, the cells were resuspended in the $1 \times$ binding buffer. After annexin V-FITC $(5 \mu \mathrm{L})$ and PI $(7 \mu \mathrm{L})$ were added to each group, samples were incubated for $15 \mathrm{~min}$ at room temperature. Cell apoptosis analysis was detected with a flow cytometry (Cytoflex, Beckman, Coulter, CA, USA).

On the other hand, cell cycle analysis was performed using PI staining cell cycle detection kit (Meilunbio, Dalian, China). Followed by centrifugation at $1000 \mathrm{~g}$ for $5 \mathrm{~min}$, the cells were resuspended in the ice-cold buffer. After fixed with $75 \%$ ice-cold ethanol at $4^{\circ} \mathrm{C}$ overnight, the cells were stained with PI staining buffer at $37^{\circ} \mathrm{C}$ for $30 \mathrm{~min}$ from light. Cell cycle analysis was also detected with a flow cytometry (Cytoflex, Beckman, Coulter, CA, USA).

2.8. Wound Healing Assay. Wound healing assay was taken to measure the capacity of $20(S)$ - Rh2 in A549 cells migration and invasion. A549 cells were seeded in 6-well plates at a density of $1 \times 10^{6}$ cells per well; after the cells grew to a monolayer, a pipette tip $(200 \mu \mathrm{L})$ was used to scraped the monolayer to create an artificial wound field. Then, cells were incubated with different concentrations $(15,22$, and $35 \mu \mathrm{M})$ of $20(S)-\mathrm{Rh} 2$. DMSO was used as a blank control. Photographs were taken at $0,6,12$, and $24 \mathrm{~h}$ using an inverted microscope (Olympus, Tokyo, Japan). The surface areas of cell wound were quantified by ImageJ software (National Institute of Health, Bethesda, MD, USA).

2.9. Molecular Docking. Based on the structure of EGFRerlotinib complex (PDB 1 M17), molecular docking was performed to predict the possible binding conformation of 20(S)-Rh2 with EGFR [21]. The cocrystallized ligand erlotinib as well as all water molecules were dislodged using Chimera 1.11 to complete the preparation of EGFR structure. The hydrogen atoms were added using AutoDockTools-
1.5.6 [22]. The structure of $20(S)$-Rh2 was generated using GaussView 5.0 and then was subject to the energy minimization using Gaussian 09 W [23]. Afterward, 20(S)-Rh2 was docked to the binding pocket of EGFR with a Lamarckian genetic algorithm using AutoDockTools-1.5.6. The grid box was generated at the center of the cocrystallized ligand erlotinib. Taken together with the above docking parameters, 10 independent calculations were performed and the conformation of $20(S)$-Rh2 with lowest binding energy was visualized using PyMOL.

2.10. Molecular Dynamics Simulation. Based on the optimal conformation of $20(S)$-Rh2 obtained from molecular docking, a 20 ns molecular dynamics simulation was performed using GROMACS 2019 [24]. The topologies of EGFR and 20(S)Rh2 were developed using CHARMM36 all-atom force field and CGenFF server, respectively [25, 26]. Afterward, the EGFR-20(S)-Rh2 complex was placed in a cubic box filling with water. The whole system was appended with counterion to obtain electrostatic neutrality. Energy minimization was completed followed with constant NVT (number of particles-volume-temperature) and constant NPT (number of particles-pressure-temperature) equilibration procedure for $0.1 \mathrm{~ns}$. With the desired temperature and pressure, the position restraint on the system was released. On this basis, molecular dynamics simulation was run and the data was collected to calculate the root mean squared deviation (RMSD) values.

2.11. Statistical Analysis. All experiments were performed independently at least three times, and all the data were expressed as mean \pm standard error of measurement (SEM). Analysis of variance (ANOVA) was used to assess the statistical significance of experimental manipulations. The difference compared with the control group was considered statistically significant at ${ }^{*} p<0.05,{ }^{* *} p<0.01$, and ${ }^{* * *} p<0.001$.

\section{Results and Discussion}

3.1. 20(S)-Rh2 Inhibited the Activity of EGFR Kinase. HTRF assay is a homogeneous time-resolved assay that generates a signal by fluorescence resonance energy transfer (FRET) between the donor and acceptor molecules [27]. The donor is a $\mathrm{Eu}^{3+}$ caged in the polycyclic cryptate, while the acceptor 


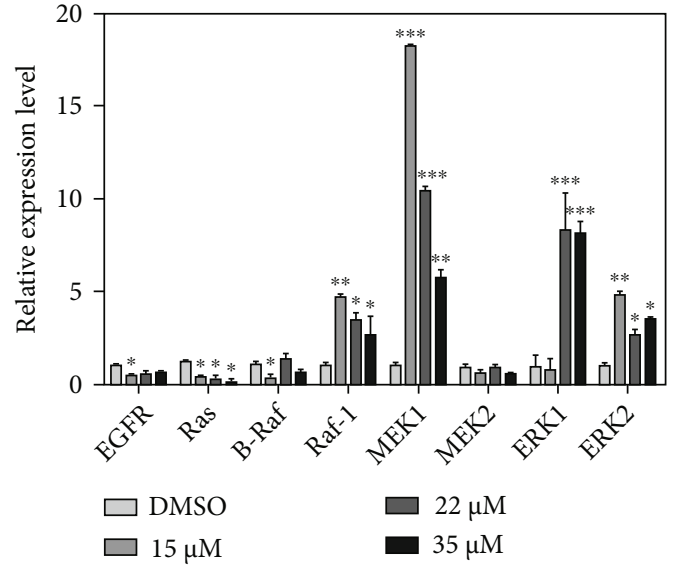

(a)

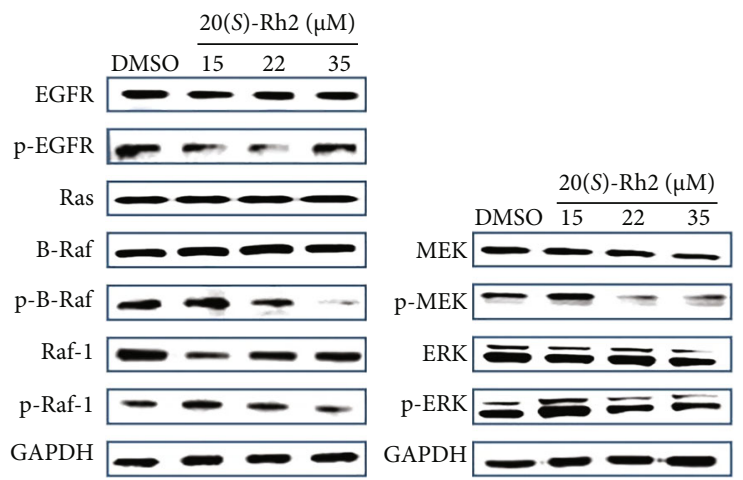

(b)

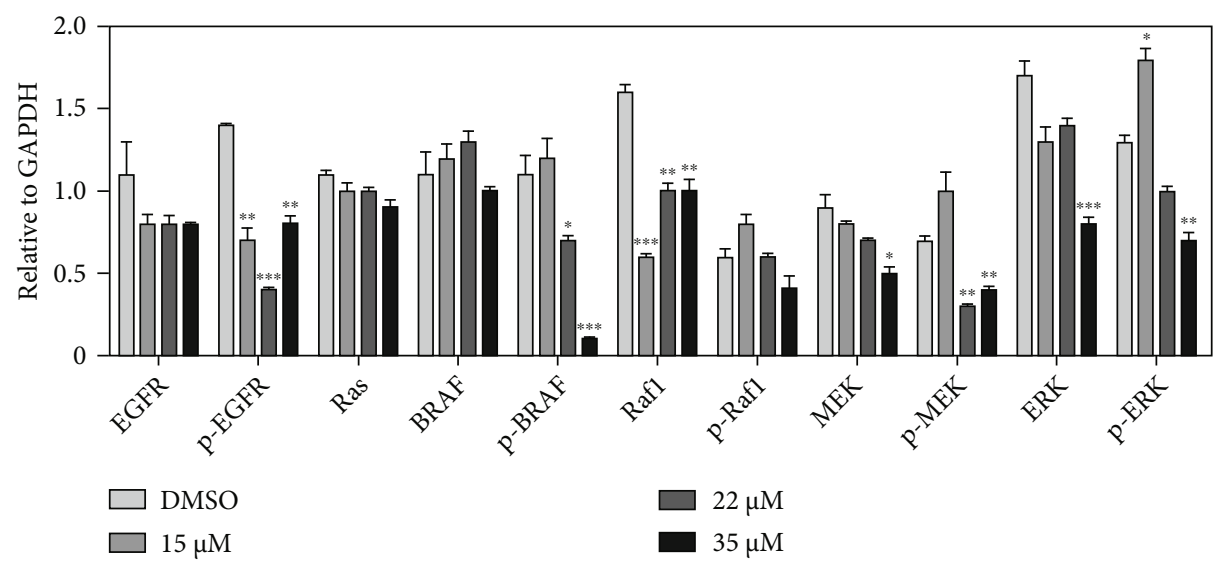

(c)

FIgURE 2: 20(S)-Rh2 regulated the EGFR-MAPK pathway in A549 cells. (a) Quantitative real-time PCR and (b) western blot analysis of $20(S)$-Rh2 to EGFR-MAPK pathway. (c) Quantitative analyses of the levels of EGFR-MAPK pathway related proteins compared to GAPDH. ${ }^{*} p<0.05,{ }^{* *} p<0.01$, and ${ }^{* * *} p<0.001$.

is a streptavidin-XL665 labeled protein. Laser excitation of the donor at $337 \mathrm{~nm}$ leads to the transfer of energy to the acceptor at $620 \mathrm{~nm}$ when they are in the close proximity, resulting in the emission of light over a prolonged period of milliseconds at $665 \mathrm{~nm}$ [28]. In this assay, HTRF was performed to analyze the effect of $20(S)$-Rh2 on EGFR kinase activity. 20(S)-Rh2 altered EGFR kinase activity in a dosedependent manner, and the inhibitory curve was presented in Figure 1(a). The $\mathrm{IC}_{50}$ of $20(\mathrm{~S})$ - Rh 2 on EGFR kinase activity was determined to be $2.78 \pm 0.16 \mu \mathrm{M}$ with the average of at least three independent experiments. The data demonstrated that 20(S)-Rh2 inhibited the activity of EGFR kinase.

3.2. Effect of 20(S)-Rh2 on the Inhibition of A549 Cell Proliferation. Exponentially growing A549 cells were seeded continuously in the presence or absence of different concentrations of $20(S)-\mathrm{Rh} 2$. The effect of $20(S)-\mathrm{Rh} 2$ on cell growth was assessed by the MTT assay. As shown in Figure 1(b), 20(S)-Rh2 inhibited the growth of A549 cells in a dosedependent manner. DMSO was used as a blank control. The $20 \%, 40 \%$, and $60 \%$ inhibitory concentrations $\left(\mathrm{IC}_{20}\right.$, $\mathrm{IC}_{40}$, and $\mathrm{IC}_{60}$ ) of $20(S)-\mathrm{Rh} 2$ on A549 cells were 15, 22, and $35 \mu \mathrm{M}$, respectively.
3.3. 20(S)-Rh2 Regulated the EGFR-MAPK Pathway in A549 Cells. To investigate whether $20(S)$-Rh2 regulated the MAPK pathway through affecting the expressions of EGFR, the changes in gene expressions and protein contents were determined by quantitative real-time PCR and western blot analysis, respectively. As shown in Figure 2(a), after the treatment of 20(S)-Rh2, the gene expression of EGFR was all downregulated. Similarly, the protein contents of totalEGFR and phospho-EGFR were all downregulated after the treatment of $20(S)-\mathrm{Rh} 2$ compared to the DMSO-treated group (Figures 2(b) and 2(c)). Combined with the result of HTRF, 20(S)-Rh2 could inhibit the phosphorylation of EGFR. After the treatment of $20(S)$-Rh2, the gene expressions of Ras and BRAF were downregulated (Figure 2(a)); the protein content of Ras was also slightly downregulated; however, the protein content of BRAF was firstly upregulated then downregulated. Especially, the protein content of phospho-BRAF was significantly downregulated at the concentrations of $35 \mu \mathrm{M}$ (Figure 2(c)). The gene expression of Raf1 was upregulated after the treatment of 20(S)-Rh2; however, the protein content of Rafl was significantly downregulated (Figure 2(c)). Apart from the gene expression of $M E K 2$ downregulated, the gene expressions of $M E K 1$, 


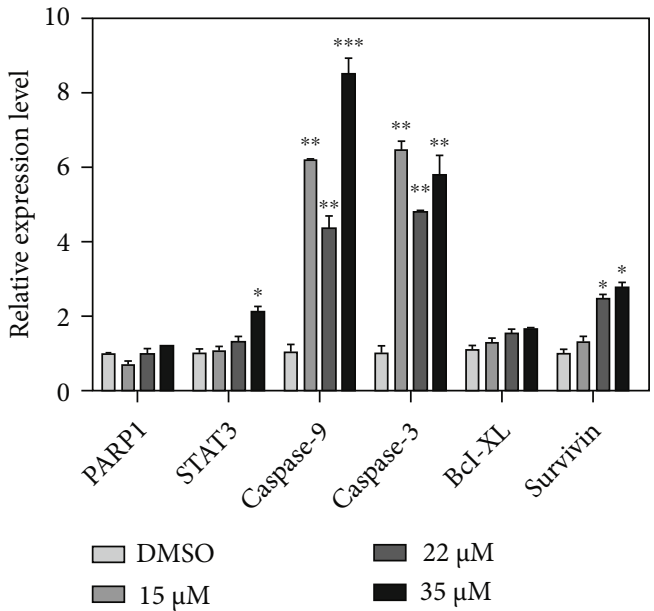

(a)

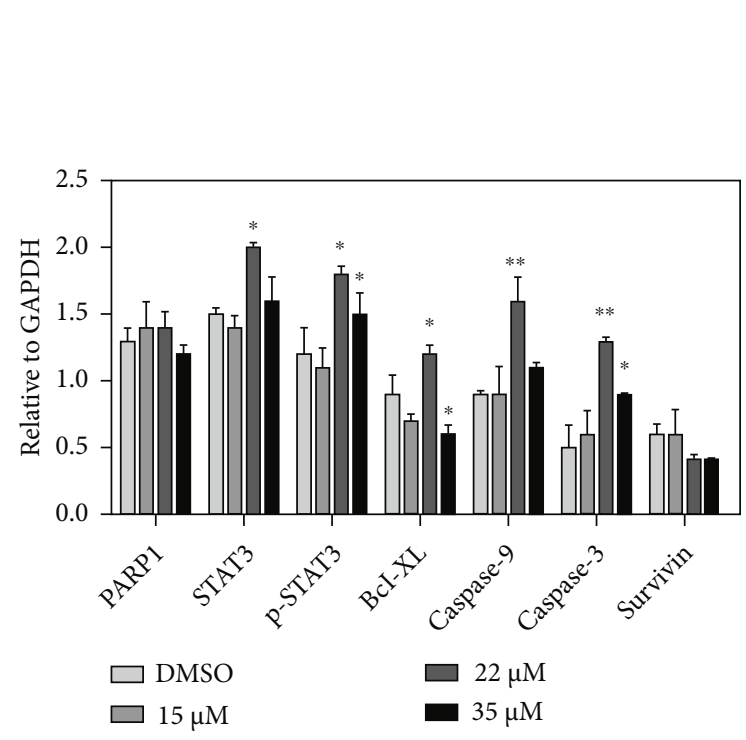

(c)

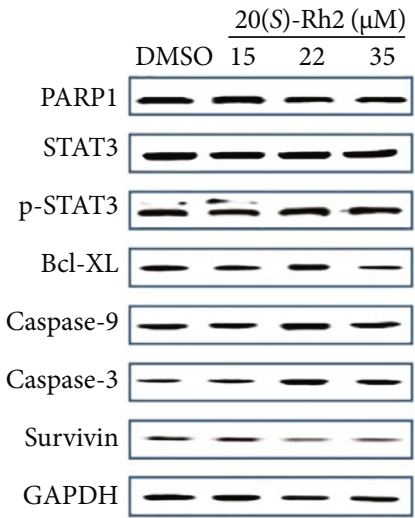

(b)
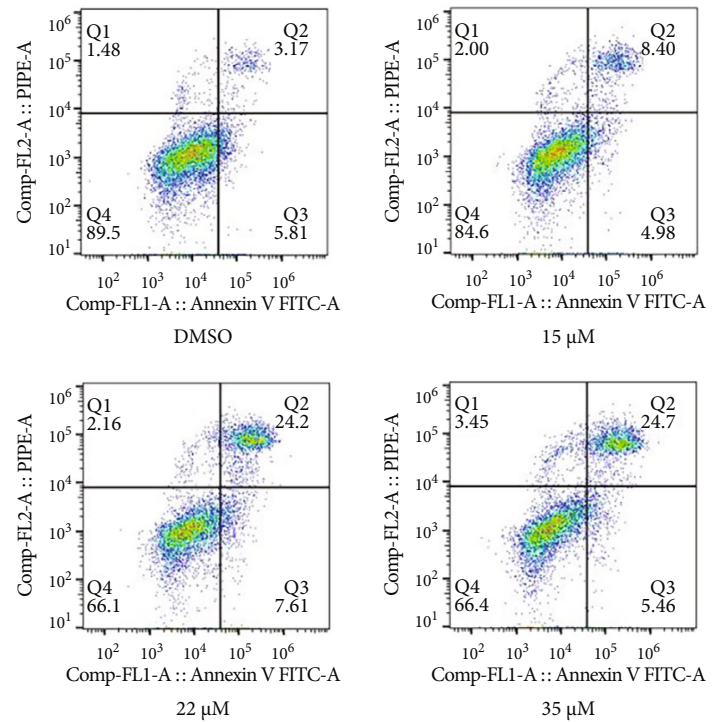

(d)

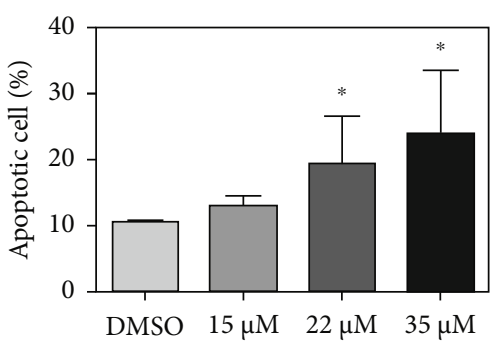

(e)

FIgURE 3: 20(S)-Rh2 promoted the apoptosis of A549 cells. The effect of 20(S)-Rh2 on A549 cells apoptosis was evaluated by (a) quantitative real-time PCR and (b) western blot analysis. (c) Quantitative analyses of the levels of cell apoptosis-related proteins compared to GAPDH. The results of (d) flow cytometry and (e) apoptotic cells of each group. ${ }^{*} p<0.05,{ }^{* *} p<0.01$, and ${ }^{* * *} p<0.001$.

$E R K 1$, and ERK2 were all significantly upregulated after the treatment of 20(S)-Rh2 (Figure 2(a)). As for the protein contents, phospho-MEK and phospho-ERK were upregulated at $15 \mu \mathrm{M}$ and then downregulated at 22 and $35 \mu \mathrm{M}$ (Figure 2(c)); total-MEK and total-ERK were downregulated (Figure 2(c)). Taken together, the results indicated that
20(S)-Rh2 inhibited the activation of EGFR to regulate the MAPK pathway leading to suppress A549 cells growth.

3.4. 20(S)-Rh2 Promoted the Apoptosis of A549 Cells. PARP1 is the first member of the PARP family that acts as a DNA damage sensor [29]. After binding on DNA damaged 


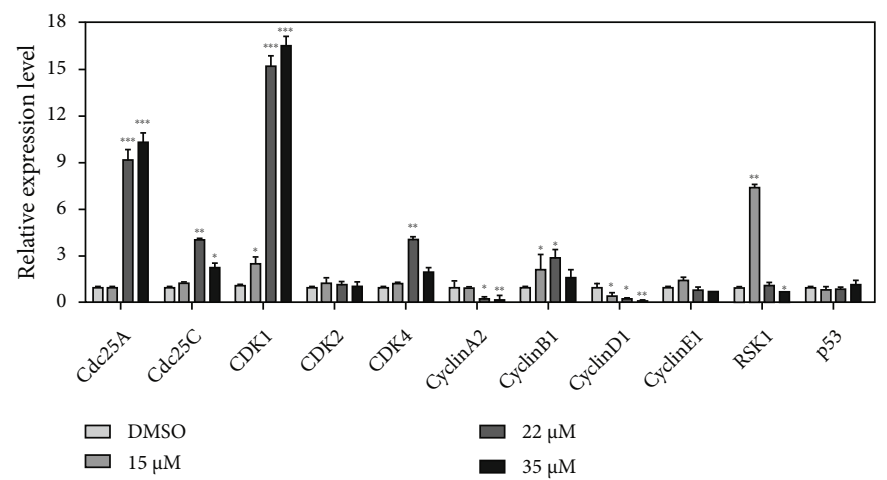

(a)

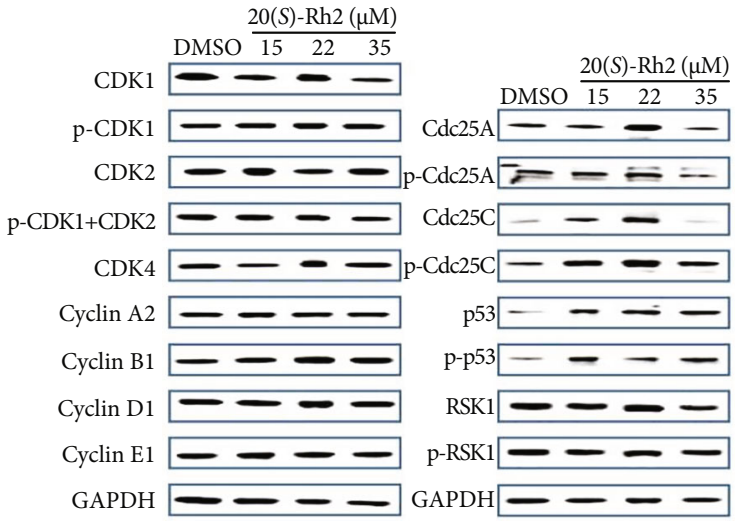

(b)

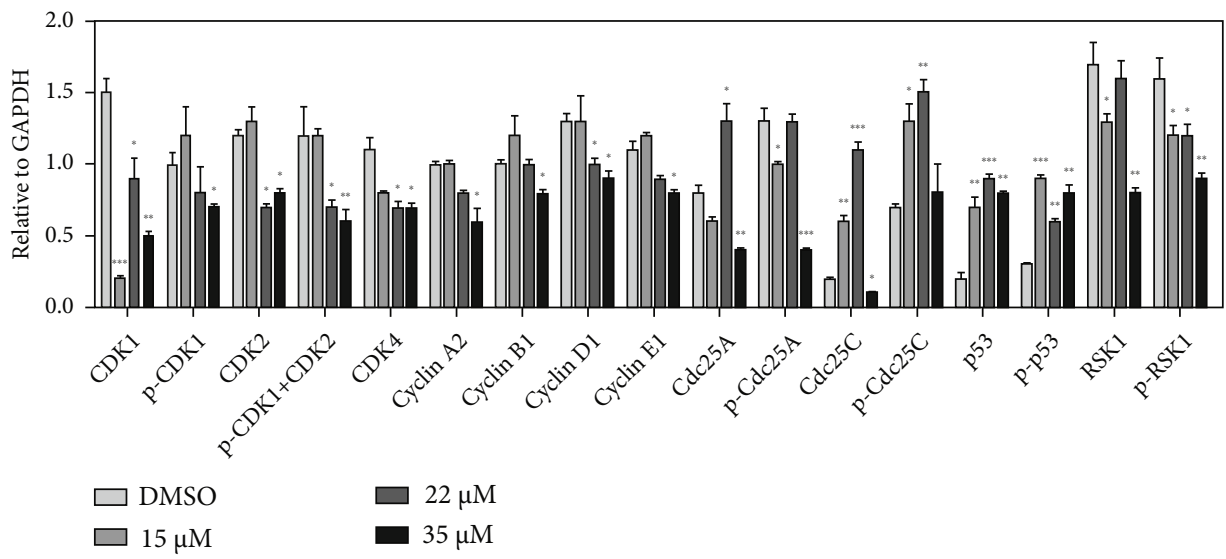

(c)
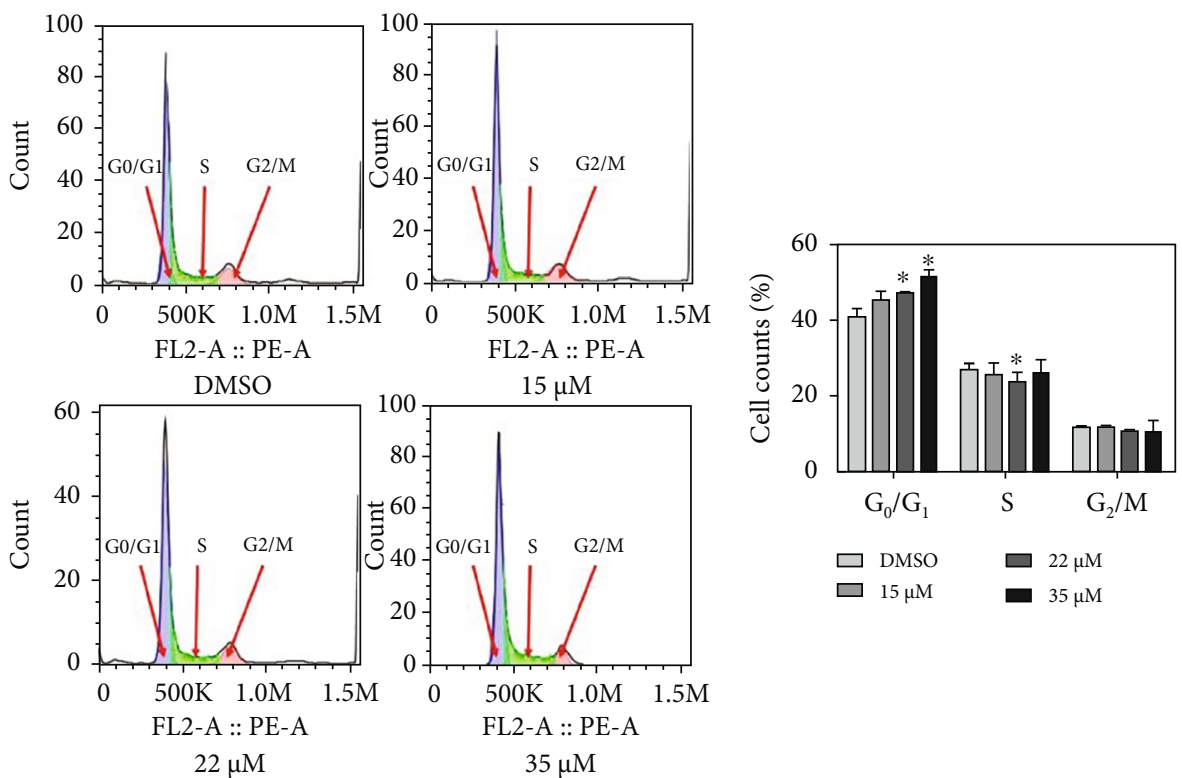

(d)

(e)

Figure 4: 20(S)-Rh2 blocked A549 cells cycle at $\mathrm{G}_{0} / \mathrm{G}_{1}$ phase. The effect of $20(\mathrm{~S})$ - $\mathrm{Rh} 2$ on A549 cells cycle was evaluated by (a) quantitative real-time PCR and (b) western blot analysis. (c) Quantitative analyses of the levels of cell cycle-related proteins compared to GAPDH. The results of (d) flow cytometry and (e) cell counts of each group. ${ }^{*} p<0.05,{ }^{* *} p<0.01$, and ${ }^{* * *} p<0.001$.

structures, PARP1 serves as a survival factor and recruits repair enzymes [30]. Caspases cleave various proteins that are necessary for the cell survival and function during the apoptosis phase [31]. PARP1 protein is cleaved by caspase3 and caspase-7 to retain basal enzymatic activity without to be stimulated by DNA damage $[32,33]$. Through this 


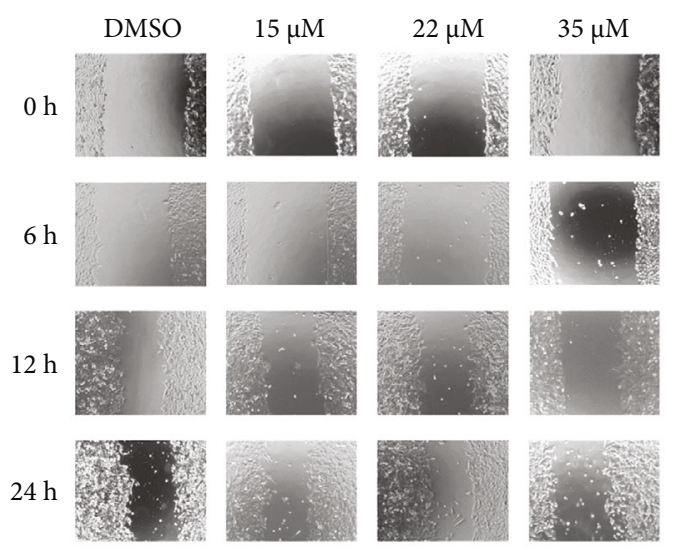

(a)

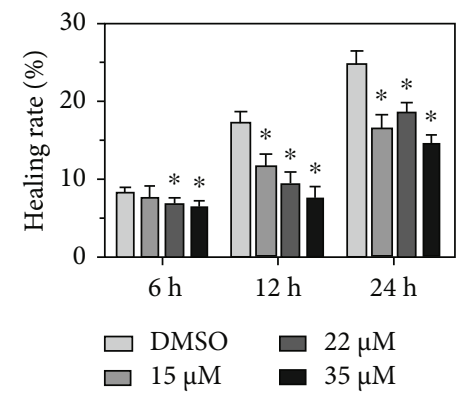

(b)

FIGURE 5: 20(S)-Rh2 reduced A549 cell migration. (a) The wound healing assay was performed on A549 cells after the treatment of 20(S)Rh2. (b) Healing rate of each group. DMSO was used as control. ${ }^{*} p<0.05$.

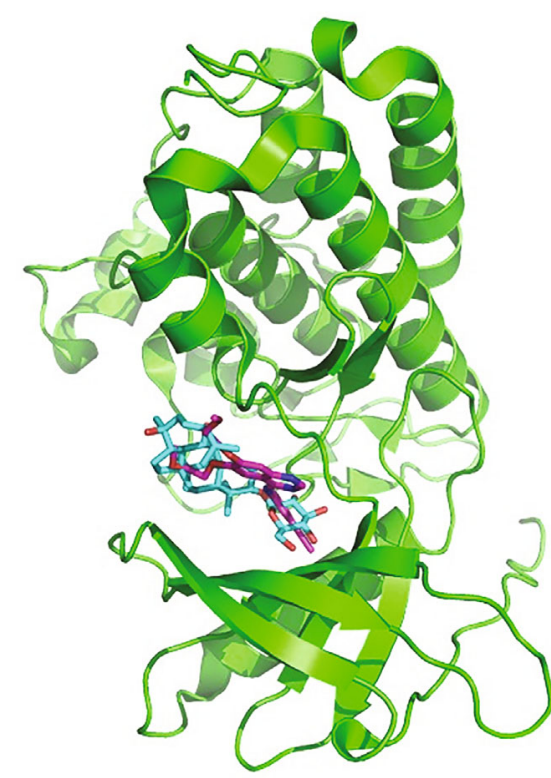

(a)

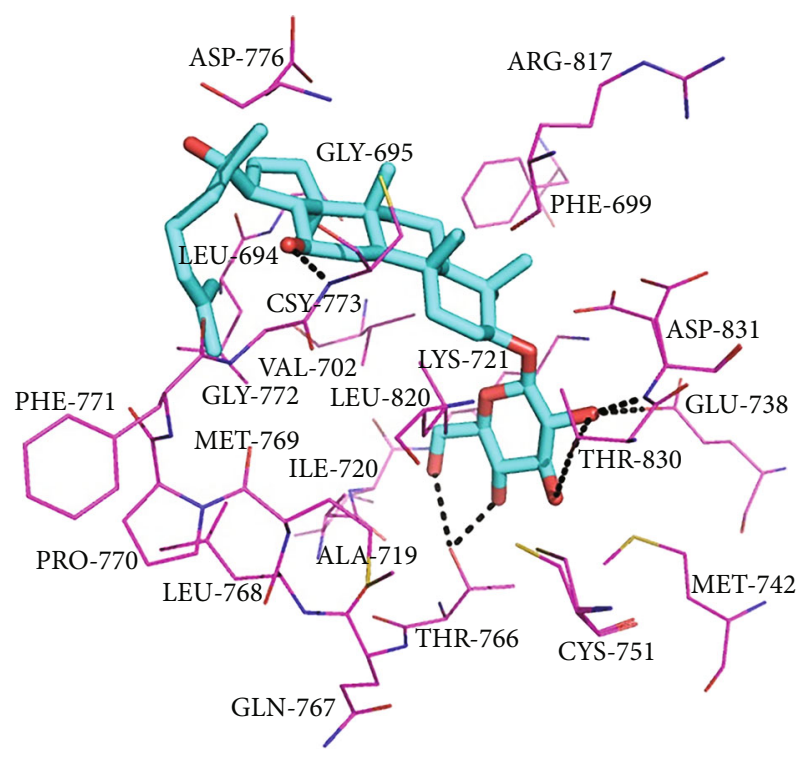

(b)

Figure 6: The result of molecular docking. (a) Docked 20(S)-Rh2 (cyan sticks) and cocrystallized ligand erlotinib (magenta sticks) in the binding pocket of EGFR (green cartoon). (b) Amino acid residues (magenta lines) of EGFR that lie within $4 \AA$ away from $20(S)$-Rh2 (cyan sticks). The hydrogen bonds were shown as black dotted lines.

process, the cleavage of PARP1 may help to induce cells to the apoptotic pathway. As shown in Figure 3(a), after the treatment of 20(S)-Rh2, although the gene expression of $P A R P 1$ was nearly unchanged, the protein content of PARP1 was firstly upregulated then downregulated at $35 \mu \mathrm{M}$ (Figures 3(b) and 3(c)) to induce A549 cell apoptosis.

The caspase cascade system plays a crucial role in the induction, transduction, and amplification of cell intracellular apoptotic signals $[34,35]$. Once caspases activated, the cells are dismantled by selectively cleaving key proteins. Caspase-9 is activated after cytochrome c (Cyt c) was released; then, effector caspases and Bid are activated to remodel the mitochondria [36]. However, the process of cell death is promoted by caspase- 3 , which is the primary execu- tioner of apoptosis. In this work, as for the caspase-9 and caspase- 3 , their gene expressions were significantly upregulated after the treatment of 20(S)-Rh2; meanwhile, their protein contents were also upregulated, especially at $22 \mu \mathrm{M}$ (Figure 3(c)). As a member of the inhibitor of apoptosis family, survivin expressed in various human cancers may induce evasion from aberrant mitotic and apoptosis progression. The treatment of $20(S)-\mathrm{Rh} 2$ downregulated the protein content of survivin at 22 and $35 \mu \mathrm{M}$ (Figure 3(c)) to induce A549 cell apoptosis.

Annexin V-FITC/PI staining was performed on the A549 cells, and the fluorescence was recorded using flow cytometry (Supplementary Figure S1). After the treatment of 20(S)-Rh2, the number of necrotic cells at Q1 and early apoptotic cells at 


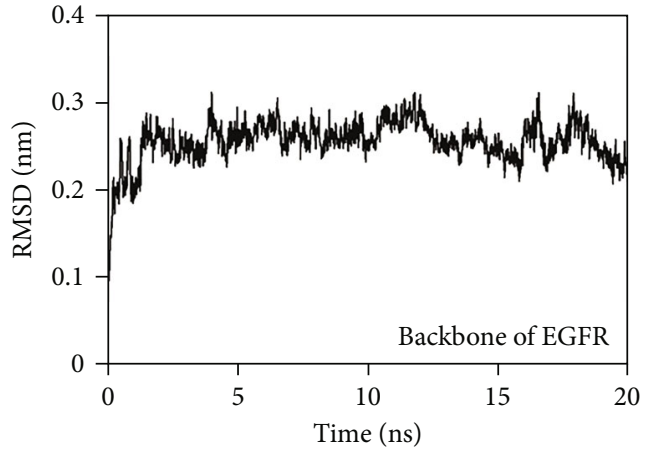

(a)

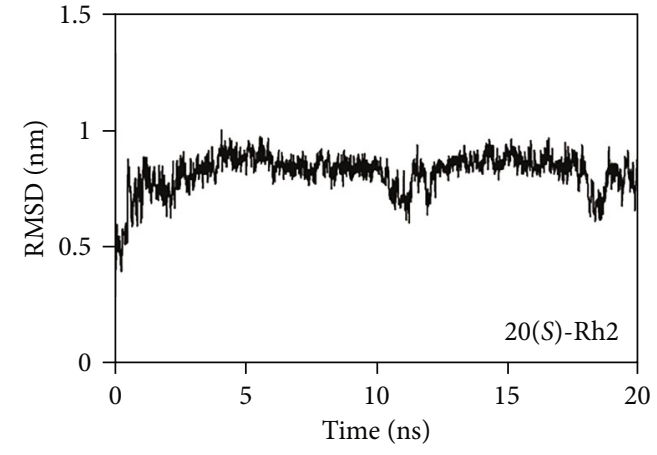

(b)

Figure 7: Variations of root mean square deviation (RMSD) values for the (a) backbone of EGFR and (b) $20(S)$-Rh2 during a $20 \mathrm{~ns}$ molecular dynamics simulation.

Q3 changed little. However, the number of late apoptotic cells at Q2 significantly increased, especially at 22 and $35 \mu \mathrm{M}$ (Figure 3(d)). The total apoptotic cells also increased in a dose-dependent manner (Figure 3(e)). Taken the results together, 20(S)-Rh2 could promote the apoptosis of A549 cells.

3.5. 20(S)-Rh2 Blocked A549 Cell Cycle at $G_{0} / G_{1}$ Phase. As key regulators of the cell cycle, cell division cycle 25 (Cdc25) phosphatase family mainly contain Cdc25A, $\mathrm{Cdc} 25 \mathrm{~B}$, and Cdc25C [37]. Although sharing the common biochemical mechanism of action, members of Cdc25 phosphatase family have unique characteristics and play specific roles in the cell cycle regulation [38]. Cdc25A plays a crucial role at the transition of $G_{1}$ to $S$ phase [39]. Cdc25B is activated during $S$ phase and induces the activation of cyclindependent protein kinase 1- (CDK1-) cyclin B in the cytoplasm [40]. Then, activated CDK1-cyclin B phosphorylates and activates $\mathrm{Cdc} 25 \mathrm{C}$ resulting in a positive feedback mechanism and entering into mitosis [41]. As shown in Figure 4(a), after the treatment of $20(S)$-Rh2, although the gene expression of $C d c 25 A$ was upregulated at high concentrations, the protein contents of total-Cdc25A and phosphoCdc25A were initially upregulated and then downregulated at $35 \mu \mathrm{M}$ (Figures $4(\mathrm{~b})$ and $4(\mathrm{c})$ ). Thus, high concentrations of $20(S)$-Rh2 could downregulate the expression of Cdc25A to inhibit the transition of $G_{1}$ to $S$ phase.

The phosphorylation of CDKs promotes the process of cell cycle. CDKs are initially activated by the combination of cyclin subunits, as well as the phosphorylation on the threonine residue in a conserved amino-acid sequence [42]. After cyclin E complexed with CDK2, this complex entries cell cycle into $S$ phase and induces the initiation of DNA replication [43]. Subsequently, cyclin A is expressed at the boundary of $G_{1}$ to $S$ phase and forms complex with CDK2 [44]. Then, the complex of cyclin A-CDK2 is necessary for both $S$ phase transition and DNA replication [45]. It is determined that after the treatment of $20(S)$ - Rh2, the gene expressions of cyclin A, cyclin E, and CDK2 were all downregulated, especially for cyclin $A$ (Figure 4(a)). Furthermore, the protein contents of cyclin A, cyclin E, and CDK2 were initially upregulated and then downregulated at high concentrations (Figure 4(c)). The downregulated contents of cyclin A, cyclin $\mathrm{E}$, and $\mathrm{CDK} 2$ inhibited the formation of cyclin A-CDK2 and cyclin E-CDK2 to block cell cycle at $\mathrm{G}_{0} / \mathrm{G}_{1}$ phase.

As a transcription factor, p53 is highly inducible by various stress signals, such as oncogene activation, DNA damage, and nutrient deprivation [46]. Activation of the p53 can lead to cell cycle arrest and cell apoptosis [47]. The crucial mechanism of p53-mediated arrest is the transcriptional downregulation of many cell cycle-related genes [41]. As shown in Figure 4(c), after the treatment of $20(S)-\mathrm{Rh} 2$, the protein contents of total-p53 and phospho-p53 were all significantly upregulated to arrest cell cycle.

PI staining was performed on the A549 cells, and the fluorescence was recorded using flow cytometry (Supplementary Figure S2). After the treatment of 20(S)-Rh2, the number of cell counts at $G_{0} / G_{1}$ phase was increased in a dose-dependent manner. Meanwhile, the number of cell counts at $S$ or $G_{2} / M$ phase was decreased compared to the DMSO control groups (Figures 4(d) and 4(e)). Taken the results together, 20(S)-Rh2 could block A549 cell cycle at $\mathrm{G}_{0} / \mathrm{G}_{1}$ phase.

3.6. 20(S)-Rh2 Reduced A549 Cell Migration. The inhibition activity of $20(S)-\mathrm{Rh} 2$ on A549 cell migration was assessed by cell wound healing assay [48]. Photomicrographs were taken at $0,6,12$, and $24 \mathrm{~h}$ after wounding (Figure 5(a)). The DMSO-treated group was used as a control. As a percentage of the initial wound area, the remaining cell-free area was taken as an index of wound healing $[49,50]$. In the DMSO control group, about $8 \%$ of the wound area healed at $6 \mathrm{~h}$ after wounding compared to the $0 \mathrm{~h}$. Meanwhile, the wound area of the $20(S)$-Rh2-treated group significantly healed at $22 \mu \mathrm{M}(6.8 \%)$ and $35 \mu \mathrm{M}(6.4 \%)$. After the treatment of $20(S)-\mathrm{Rh} 2$ at $12 \mathrm{~h}$, the migration rate of the transfected A549 cells significantly reduced in a dosedependent manner. The healing rate reduced to $7.5 \%$ at $35 \mu \mathrm{M}$ of $20(S)-\mathrm{Rh} 2$ compared to the $17.3 \%$ of the DMSOtreated group. Interestingly, the healing rate also significantly reduced at $24 \mathrm{~h}$ indicated that $20(S)-\mathrm{Rh} 2$ inhibited the A549 cell mobility at $24 \mathrm{~h}$. In general, after the treatment of $20(S)-\mathrm{Rh} 2$, the closure of the wounded area healed slowly (Figure 5(b)). Therefore, 20(S)-Rh2 could reduce A549 cell migration. 
3.7. Possible Binding Conformation of 20(S)-Rh2 with EGFR. The result of molecular docking suggested that 20(S)-Rh2 was accommodated in the binding pocket of EGFR, adopting a similar binding conformation with that of co-crystallized ligand erlotinib (Figure 6(a)). As shown in Figure 6(b), 20(S)-Rh2 made hydrophobic interactions with 11 amino acid residues, including Leu694, Phe699, Val702, Ala719, Ile720, Met742, Leu768, Met769, Pro770, Phe771, and Leu820. The side chain sugar moiety attached at C-3 position (Figure 1(a)) formed hydrogen bonds with residues Glu738, Thr766, Thr830, and Asp831. Meanwhile, the hydroxyl group at primary ring also formed a hydrogen bond with residue Cys773. Collectively, these findings suggested that both hydrophobic interactions and hydrogenbonding interactions could contribute to stabilize the EGFR-20(S)-Rh2 binding.

3.8. Binding Stability of the EGFR-20(S)-Rh2 Complex. In this work, a $20 \mathrm{~ns}$ molecular dynamics simulation was performed to explore the binding stability of the EGFR-20(S)Rh2 complex. The backbone of EGFR basically kept stable during the whole simulation process with the average RMSD values of $0.25 \pm 0.03 \mathrm{~nm}$ (Figure $7(\mathrm{a})$ ), indicating that $20(S)$ Rh2 caused little conformational change toward EGFR. Conversely, 20(S)-Rh2 underwent a relatively severe disturbance during $20 \mathrm{~ns}$ molecular dynamics simulation, with the average RMSD values of $0.82 \pm 0.08 \mathrm{~nm}$ (Figure $7(\mathrm{~b})$ ). As shown in Figures 1(a) and 6(a), the side chain sugar moiety of $20(S)-\mathrm{Rh} 2$ was too flexible to be fixed at the active site of EGFR, which might be responsible for the structural disturbance of $20(S)-\mathrm{Rh} 2$.

\section{Conclusion}

HTRF assay was taken to confirm that 20(S)-Rh2 significantly inhibited the activity of EGFR kinase. The changes in gene expressions and protein contents demonstrated that 20(S)-Rh2 regulated the EGFR-MAPK pathway to inhibit A549 cell proliferation. The findings of the present study suggested that $20(S)$-Rh2 could promote cell apoptosis, block cell cycle, and reduce cell migration of A549 cells. The result of molecular docking suggested that both hydrophobic interactions and hydrogen-bonding interactions could contribute to stabilize the EGFR-20(S)-Rh2 binding. Further exploration of their binding stability was also performed by molecular dynamics simulation. Collectively, these findings suggested that $20(S)-\mathrm{Rh} 2$ might serve as a potential EGFR tyrosine kinase inhibitor.

\section{Data Availability}

The data used to support the findings of this study are available from the corresponding author upon request.

\section{Conflicts of Interest}

The authors declare no conflict of interest.

\section{Authors' Contributions}

Yuan Liang, Jie Zhang, and Tiehua Zhang conceived and designed the experiments. Yuan Liang, Jingqi Zhao, and Haoyang Zou performed the experiments. Yuan Liang analyzed the data and wrote the manuscript. Tiehua Zhang and Jie Zhang revised the manuscript. All authors read and approved the final manuscript.

\section{Acknowledgments}

This work was supported by the National Natural Science Foundation of China (31972160 and 31871717), the Science and Technology Development Project Foundation of Jilin Province (20200201204JC), and the Ph.D. Interdisciplinary Research Funding Project of Jilin University (101832020DJX054).

\section{Supplementary Materials}

Supplementary Figure S1: related to Figure 3. Cell apoptosis assay by flow cytometry. Supplementary Figure S2: related to Figure 4. Cell cycle assay by flow cytometry. (Supplementary Materials)

\section{References}

[1] F. Li, S. Zhao, T. Guo, J. Li, and C. Gu, "The nutritional cytokine leptin promotes NSCLC by activating the PI3K/AKT and MAPK/ERK pathways in NSCLC cells in a paracrine manner," BioMed Research International, vol. 2019, Article ID 2585743, 8 pages, 2019.

[2] A. M. Krasinskas, "EGFR signaling in colorectal carcinoma," Pathology Research International, vol. 2011, Article ID 932932, 6 pages, 2011.

[3] Y. Liang, T. Zhang, and J. Zhang, "Natural tyrosine kinase inhibitors acting on the epidermal growth factor receptor: their relevance for cancer therapy," Pharmacological Research, vol. 161, article 105164, 2020.

[4] Y. Liang, T. Zhang, L. Ren et al., "Cucurbitacin IIb induces apoptosis and cell cycle arrest through regulating EGFR/ MAPK pathway," Environmental Toxicology and Pharmacology, vol. 81, article 103542, 2021.

[5] Q. Wei, J. Li, Y. Zhan et al., "Enhancement of glucose homeostasis through the PI3K/Akt signaling pathway by dietary with Agaricus blazei Murrill in STZ-induced diabetic rats," Food Science and Nutrition, vol. 8, no. 2, pp. 1104-1114, 2020.

[6] X. Wei, B. Zhang, X. Liang et al., "Higenamine alleviates allergic rhinitis by activating AKT1 and suppressing the EGFR/ JAK2/c-JUN signaling," Phytomedicine, vol. 86, article 153565, 2021.

[7] G. J. Weiss, L. T. Bemis, E. Nakajima et al., "EGFR regulation by microRNA in lung cancer: correlation with clinical response and survival to gefitinib and EGFR expression in cell lines," Annals of Oncology, vol. 19, no. 6, pp. 1053-1059, 2008.

[8] C. Peraldo-Neia, G. Migliardi, M. Mello-Grand et al., "Epidermal growth factor receptor (EGFR) mutation analysis, gene expression profiling and EGFR protein expression in primary prostate cancer," BMC Cancer, vol. 11, no. 1, p. 31, 2011.

[9] Y. Liang, T. Zhang, S. Jing et al., "20(S)-Ginsenoside Rg3 inhibits lung cancer cell proliferation by targeting EGFR- 
mediated Ras/Raf/MEK/ERK pathway," The American Journal of Chinese Medicine, vol. 49, no. 3, pp. 753-765, 2021.

[10] T. Zhang, Y. Liang, and J. Zhang, "Natural and synthetic compounds as dissociated agonists of glucocorticoid receptor," Pharmacological Research, vol. 156, article 104802, 2020.

[11] J. Zhang, P. Pavek, R. Kamaraj, L. Ren, and T. Zhang, "Dietary phytochemicals as modulators of human pregnane $\mathrm{X}$ receptor," Critical Reviews in Food Science and Nutrition, pp. 123, 2021.

[12] T. Zhang, S. Zhong, L. Hou et al., "Computational and experimental characterization of estrogenic activities of $20(S, R)$ protopanaxadiol and 20(S,R)-protopanaxatriol," Journal of Ginseng Research, vol. 44, no. 5, pp. 690-696, 2020.

[13] T. Zhang, S. Zhong, T. Li, and J. Zhang, "Saponins as modulators of nuclear receptors," Critical Reviews in Food Science and Nutrition, vol. 60, no. 1, pp. 94-107, 2020.

[14] L. Ren, J. Zhang, and T. Zhang, "Immunomodulatory activities of polysaccharides from Ganoderma on immune effector cells," Food Chemistry, vol. 340, article 127933, 2021.

[15] H. Li, N. Huang, W. Zhu et al., "Modulation the crosstalk between tumor-associated macrophages and non-small cell lung cancer to inhibit tumor migration and invasion by ginsenoside Rh2," BMC Cancer, vol. 18, no. 1, p. 579, 2018.

[16] X. Sun, P. Zhao, H. Li, Y. Liu, T. Wang, and Y. Cheng, "Ginsenoside Rh2 inhibits glycolysis through the STAT3/c-MYC axis in non-small-cell lung cancer," Journal of Oncology, vol. 2021, Article ID 9715154, 12 pages, 2021.

[17] Y. Chen, Y. Zhang, W. Song, Y. Zhang, X. Dong, and M. Tan, "Ginsenoside Rh2 improves the cisplatin anti-tumor effect in lung adenocarcinoma A549 cells via superoxide and PD-L1," Anti-Cancer Agents in Medicinal Chemistry, vol. 20, no. 4, pp. 495-503, 2020.

[18] J. Zhao, T. Zhang, Y. Liang, H. Zou, and J. Zhang, "Inhibitory activities of $20(R, S)$-protopanaxatriol against epidermal growth factor receptor tyrosine kinase," Food and Chemical Toxicology, vol. 155, article 112411, 2021.

[19] Y. Liang, J. Zhao, H. Zou, J. Zhang, and T. Zhang, "In vitro and in silico evaluation of EGFR targeting activities of curcumin and its derivatives," Food \& Function, vol. 12, no. 21, pp. 10667-10675, 2021.

[20] T. Zhang, Y. Liang, P. Zuo et al., "20(S)-Protopanaxadiol blocks cell cycle progression by targeting epidermal growth factor receptor," Food and Chemical Toxicology, vol. 135, article 111017, 2020.

[21] J. Stamos, M. X. Sliwkowski, and C. Eigenbrot, "Structure of the epidermal growth factor receptor kinase domain alone and in complex with a 4 -anilinoquinazoline inhibitor," Journal of Biological Chemistry, vol. 277, no. 48, pp. 46265-46272, 2002.

[22] J. Zhang, J. Zhao, Y. Sun et al., "GR-mediated antiinflammation of $\alpha$-boswellic acid: Insights from in vitro and in silico studies," Food and Chemical Toxicology, vol. 155, article 112379, 2021.

[23] T. Zhang, Y. Liang, P. Zuo et al., "Identification of $20(R, S)$ protopanaxadiol and $20(R, S)$-protopanaxatriol for potential selective modulation of glucocorticoid receptor," Food and Chemical Toxicology, vol. 131, article 110642, 2019.

[24] J. Zhang, Y. Liang, L. Ren, and T. Zhang, "In vitro antiinflammatory potency of sanguinarine and chelerythrine via interaction with glucocorticoid receptor," eFood, vol. 1, no. 6 , pp. 392-398, 2020.
[25] L. Ren, S. Niu, Y. Sun et al., "Anti-inflammatory action of betulin and its potential as a dissociated glucocorticoid receptor modulator," Food and Chemical Toxicology, vol. 157, article 112539, 2021.

[26] Y. Leng, L. Ren, S. Niu, T. Zhang, and J. Zhang, "In vitro and in silico investigations of endocrine disruption induced by metabolites of plasticizers through glucocorticoid receptor," Food and Chemical Toxicology, vol. 155, article 112413, 2021.

[27] Z. Su, W. Tian, J. Li et al., "Biological evaluation and molecular docking of Rhein as a multi-targeted radiotherapy sensitization agent of nasopharyngeal carcinoma," Journal of Molecular Structure, vol. 1147, pp. 462-468, 2017.

[28] S. Lei, Z. He, T. Chen et al., "Long noncoding RNA 00976 promotes pancreatic cancer progression through OTUD7B by sponging miR-137 involving EGFR/MAPK pathway," Journal of Experimental and Clinical Cancer Research, vol. 38, no. 1, p. 470, 2019.

[29] Y. Wang, W. Luo, and Y. Wang, "PARP-1 and its associated nucleases in DNA damage response," DNA Repair, vol. 81, article 102651, 2019.

[30] P. T. Diamantopoulos, M. Sofotasiou, V. Papadopoulou, K. Polonyfi, T. Iliakis, and N.-A. Viniou, "PARP1-driven apoptosis in chronic lymphocytic leukemia," BioMed Research International, vol. 2014, Article ID 106713, 6 pages, 2014.

[31] M. V. Fiandalo and N. Kyprianou, "Caspase control: protagonists of cancer cell apoptosis," Experimental Oncology, vol. 34, no. 3, pp. 165-175, 2012.

[32] S. Erener, V. Pétrilli, I. Kassner et al., "Inflammasome-activated caspase 7 cleaves PARP1 to enhance the expression of a subset of NF- $\kappa \mathrm{B}$ target genes," Molecular Cell, vol. 46, no. 2, pp. 200-211, 2012.

[33] A. Flütsch, R. Ackermann, T. Schroeder et al., "Combined inhibition of caspase 3 and caspase 7 by two highly selective DARPins slows down cellular demise," The Biochemical Journal, vol. 461, no. 2, pp. 279-290, 2014.

[34] I. Chowdhury, B. Tharakan, and G. K. Bhat, "Caspases - an update," Comparative Biochemistry and Physiology Part B: Biochemistry and Molecular Biology, vol. 151, no. 1, pp. 1027, 2008.

[35] X. M. Yin, "Signal transduction mediated by Bid, a pro-death Bcl-2 family proteins, connects the death receptor and mitochondria apoptosis pathways," Cell Research, vol. 10, no. 3, pp. 161-167, 2000.

[36] M. Brentnall, L. Rodriguez-Menocal, R. L. De Guevara, E. Cepero, and L. H. Boise, "Caspase-9, caspase-3 and caspase-7 have distinct roles during intrinsic apoptosis," BMC Cell Biology, vol. 14, no. 1, p. 32, 2013.

[37] M. Donzelli and G. F. Draetta, "Regulating mammalian checkpoints through Cdc25 inactivation," EMBO Reports, vol. 4, no. 7, pp. 671-677, 2003.

[38] K. Liu, M. Zheng, R. Lu et al., "The role of CDC25C in cell cycle regulation and clinical cancer therapy: a systematic review," Cancer Cell International, vol. 20, no. 1, p. 213, 2020.

[39] L. Busino, M. Donzelli, M. Chiesa et al., "Degradation of Cdc25A by $\beta$-TrCP during $S$ phase and in response to DNA damage," Nature, vol. 426, no. 6962, pp. 87-91, 2003.

[40] A. J. Lincoln, D. Wickramasinghe, P. Stein et al., "Cdc25b phosphatase is required for resumption of meiosis during oocyte maturation," Nature Genetics, vol. 30, no. 4, pp. 446449, 2002. 
[41] K. Engeland, "Cell cycle arrest through indirect transcriptional repression by p53: I have a DREAM," Cell Death and Differentiation, vol. 25, no. 1, pp. 114-132, 2018.

[42] Y.-Y. Zhang, F. Zhang, Y.-S. Zhang et al., "Mechanism of juglone-induced cell cycle arrest and apoptosis in Ishikawa human endometrial cancer cells," Journal of Agricultural and Food Chemistry, vol. 67, no. 26, pp. 7378-7389, 2019.

[43] D. Coverley, H. Laman, and R. A. Laskey, "Distinct roles for cyclins E and A during DNA replication complex assembly and activation," Nature Cell Biology, vol. 4, no. 7, pp. 523$528,2002$.

[44] C. L. Nguyen and K. Münger, "Direct association of the HPV16 E7 oncoprotein with cyclin A/CDK2 and cyclin E/ CDK2 complexes," Virology, vol. 380, no. 1, pp. 21-25, 2008.

[45] V. Oakes, W. Wang, B. Harrington et al., "Cyclin A/Cdk2 regulates $\mathrm{Cdh} 1$ and claspin during late $S / \mathrm{G}_{2}$ phase of the cell cycle," Cell Cycle, vol. 13, no. 20, pp. 3302-3311, 2014.

[46] A. M. Kaiser and L. D. Attardi, "Deconstructing networks of p53-mediated tumor suppression in vivo," Cell Death and Differentiation, vol. 25, no. 1, pp. 93-103, 2018.

[47] A. M. Puzio-Kuter, "The role of p53 in metabolic regulation," Genes \& Cancer, vol. 2, no. 4, pp. 385-391, 2011.

[48] J. E. N. Jonkman, J. A. Cathcart, F. Xu et al., "An introduction to the wound healing assay using live-cell microscopy," Cell Adhesion \& Migration, vol. 8, no. 5, pp. 440-451, 2014.

[49] J. Zhang, Y. Wu, X. Zhao et al., "Chemopreventive effect of flavonoids from Ougan (Citrus reticulata cv. Suavissima) fruit against cancer cell proliferation and migration," Journal of Functional Foods, vol. 10, pp. 511-519, 2014.

[50] S. Guo and L. A. Dipietro, "Factors affecting wound healing," Journal of Dental Research, vol. 89, no. 3, pp. 219-229, 2010. 